\title{
Thermodynamics of Partitioning of Phenothiazine Drugs between Phosphatidylcholine Bilayer Vesicles and Water Studied by Second-Derivative Spectrophotometry
}

\author{
Shigehiko Takegami, Keisuke Kitamura, ${ }^{*}$ Tatsuya Kitade, Ai Kitagawa, and Kikuko Kawamura \\ Kyoto Pharmaceutical University; 5 Nakauchicho, Misasagi, Yamashina-ku, Kyoto 607-8414, Japan. \\ Received April 28, 2003; accepted June 25, 2003
}

The partition coefficients $\left(K_{\mathrm{p}} s\right)$ of phenothiazine drugs (trifluoperazine, triflupromazine, chlorpromazine and promazine) between phosphatidylcholine (PC) small unilamellar vesicles (SUV) and water were determined over the temperature range of $10-40^{\circ} \mathrm{C}$ by a second-derivative spectrophotometric method. The second derivative spectra of each drug solution containing various amounts of SUV showed distinct derivative isosbestic points confirming the entire elimination of the residual background signal effects of the SUV. The $K_{p}$ values were calculated from the derivative intensity change of the drugs induced by the addition of SUV to the drug buffer solutions (pH 7.4) and obtained with the R.S.D. below $10 \%(n=3)$. The van't Hoff analysis of the temperature dependence of $K_{\mathrm{p}}$ values revealed negative $\Delta H_{\mathrm{w} \rightarrow 1}$ and positive $\Delta S_{\mathrm{w} \rightarrow 1}$, suggesting an enthalpy/entropy driven mechanism for the phenothiazine partitioning. The negative $\Delta H_{\mathrm{w} \rightarrow 1}$ implies that the electrostatic interaction, positively charged alkyl amino groups of phenothiazine drugs with negatively charged phosphate groups on the surface of PC SUV, partly contributes to the partitioning. The existence of halogen atom(s) on the phenothiazine ring at position $\mathrm{C}-2$ enhanced the $K_{\mathrm{p}}$ value $\left(\mathrm{H}<\mathrm{Cl}<\mathrm{CF}_{3}\right)$. This enhancement can be accounted for by an increase in the $\Delta S_{\mathrm{w} \rightarrow \mathrm{l}}$ value $\left(\mathrm{H}<\mathrm{Cl}<\mathrm{CF}_{3}\right)$, and the $\Delta S_{\mathrm{w} \rightarrow \mathrm{l}}$ increase is considered to be enhancement of disorder in the hydrophobic acyl chain regions of PC SUV membranes derived from the phenothiazine ring insertion and thus depends on the bulkiness of the substituent. The enthalpy-entropy correlation analysis yielding a good linear relationship also suggests that the phenothiazine drugs studied have identically an enthalpy-entropy compensation mechanism for the partitioning.

Key words phenothiazine; partition coefficient; thermodynamics; second-derivative spectrophotometry; liposome

Partition coefficients of drugs between lipid bilayer vesicles (liposomes) and water provide fundamental information relating to the drug interactions with biomembranes. Of particular interests are thermodynamics of the partitioning process into lipid bilayer membranes. Most drugs usually partition into cell membrane by passive diffusion. Thus, thermodynamics can reveal not only essential driving forces of drug partitioning into lipid bilayer membranes but also helpful information for their pharmacodynamic and pharmacokinetic understanding. The thermodynamics of the drug partitioning has been studied in $n$-octanol/water systems and liposome/water systems. ${ }^{1-6)}$ However, $n$-octanol/water system is a too simplified biological model to study the drug-membrane interactions which are important for membrane transport and physiological activity of drugs. It is suggested that the partition coefficients obtained with liposome/water systems are superior to those derived from non-polar solvent/ water systems in quantitative structure-activity relationship (QSAR) applications. ${ }^{7-9)}$

By using the liposome/water system, and applying secondderivative spectrophotometry, we previously reported the partition coefficients of widely used psychotropic phenothiazine drugs, chlorpromazine and promazine. ${ }^{10)}$ Further, the effects of liposome size and cholesterol content on the phenothiazine partition coefficients in the liposome/water system were clarified. ${ }^{11)}$ In this study we examined the thermodynamics of the partitioning of trifluoperazine (TFPZ), triflupromazine (TFZ), chlorpromazine (CPZ) and promazine (PZ), into sonicated phosphatidylcholine (PC) small unilamellar vesicles (SUV) also by using second-derivative spectrophotometry. It has been recognized that the secondderivative spectrophotometry can eliminate the effect of background signals ${ }^{12,13)}$ and be usefully applicable to the determination of the partition coefficients of drugs between lipid vesicle and water ${ }^{10)}$ without the troublesome separation procedures which may disturb the equilibrium states and may cause fluctuation in the temperature of sample solutions. ${ }^{14-16)}$ The use of SUV as a biomembrane model in the thermodynamic studies is considered to be more suitable than multilamellar liposomes that have been widely used where separation procedures were employed.

\section{Experimental}

Calculation of Molar Partition Coefficients The molar partition coefficient $\left(K_{\mathrm{p}}\right)$ of phenothiazine between the PC SUV and water is defined as, ${ }^{10,17)}$

$$
K_{\mathrm{p}}=\frac{\left(\left[\mathrm{P}_{\mathrm{m}}\right] /\left[\mathrm{P}_{\mathrm{t}}\right]\right) /[\mathrm{L}]}{\left(\left[\mathrm{P}_{\mathrm{W}}\right] /\left[\mathrm{P}_{\mathrm{t}}\right]\right) /[\mathrm{W}]}
$$

where $\left[\mathrm{P}_{\mathrm{m}}\right]$ and $\left[\mathrm{P}_{\mathrm{W}}\right]$ represent the concentrations of phenothiazine in the PC bilayer of SUV and water, respectively, and $\left[\mathrm{P}_{\mathrm{t}}\right]=\left[\mathrm{P}_{\mathrm{m}}\right]+\left[\mathrm{P}_{\mathrm{W}}\right]$, and $[\mathrm{L}]$ and [W] are molar concentrations of PC in SUV and water $\left(55.3 \mathrm{~m}\right.$ at $\left.37^{\circ} \mathrm{C}\right)$, respectively.

If the background signal effect based on the PC SUV is eliminated in the second derivative spectra, the derivative intensity difference $(\Delta D)$ of phenothiazine before and after the addition of PC SUV at a specific wavelength is proportional to the concentration of phenothiazine in the PC SUV. As described in a previous paper, ${ }^{10)}$ we can get Eq. 2 from Eq. 1,

$$
\Delta D=\frac{K_{\mathrm{P}} \Delta D_{\max }[\mathrm{L}]}{[\mathrm{W}]+\mathrm{K}_{\mathrm{P}}[\mathrm{L}]}
$$

where $\Delta D_{\max }$ is the maximum $\Delta D$ value assuming all phenothiazines are partitioned in the PC SUV. The values of $K_{\mathrm{p}}$ and $\Delta D_{\max }$ can be calculated from the experimental values of [L] and $\Delta D$ by applying a non-linear leastsquares calculation to Eq. 2. The calculation was performed by a personal computer. ${ }^{10)}$

Calculations of Thermodynamic Parameters In order to calculate 
thermodynamic parameters, the molar partition coefficients, $K_{\mathrm{p}}(\mathrm{mol})$, of phenothiazine drugs are converted to the $K_{\mathrm{p}}(\mathrm{vol})$ based on the membrane and buffer volumes, i.e., $K_{\mathrm{p}}(\mathrm{mol})$ is divided by 42 as $\left.K_{\mathrm{p}}(\mathrm{mol}) / 42=K_{\mathrm{p}}(\mathrm{vol}){ }^{17}\right)$

The free energy of partitioning $\left(\Delta G_{\mathrm{w} \rightarrow 1}\right)$ is related to $K_{\mathrm{p}}(\mathrm{vol})$ by Eq. 3,

$$
\Delta G_{\mathrm{w} \rightarrow 1}=-R T \ln K_{\mathrm{p}}(\mathrm{vol})
$$

where $R$ is the gas constant $\left(8.314 \mathrm{~J} \cdot \mathrm{mol}^{-1} \mathrm{~K}^{-1}\right)$ and $T$ is the temperature in Kelvin.

The temperature dependence of partitioning was used to obtain the enthalpy $\left(\Delta H_{\mathrm{w} \rightarrow \mathrm{l}}\right)$ of the process which was determined from the slope of the van't Hoff plot of $\ln K_{\mathrm{p}}(\mathrm{vol}) v s .1 / T$ as below,

$$
\ln K_{\mathrm{p}}(\mathrm{vol})=\frac{\Delta S_{\mathrm{w} \rightarrow 1}}{R}-\frac{\Delta H_{\mathrm{w} \rightarrow \mathrm{l}}}{R T}
$$

The change in entropy $\left(\Delta S_{\mathrm{w} \rightarrow 1}\right)$ at a given temperature is calculated from Eq. 5 using the obtained values of $\Delta H_{\mathrm{w} \rightarrow 1}$ and $\Delta G_{\mathrm{w} \rightarrow 1}$ :

$$
\Delta S_{\mathrm{w} \rightarrow 1}=\frac{\Delta H_{\mathrm{w} \rightarrow 1}-\Delta G_{\mathrm{w} \rightarrow 1}}{T}
$$

Reagents TFPZ dihydrochloride, TFZ hydrochloride, CPZ hydrochloride and PZ hydrochloride (Fig. 1) were purchased from Sigma and used without further purification. The buffer used was $50 \mathrm{~mm} \mathrm{NaCl}-10 \mathrm{~mm} 4-(2-$ hydroxyethyl)-1-piperazineethanesulfonic acid (Hepes buffer, pH 7.4). Egg $\mathrm{L}-\alpha$-PC of $99 \%$ purity was purchased as a $2 \%(\mathrm{w} / \mathrm{v})$ chloroform solution from Avanti Polar-Lipids Inc. (Alabaster, AL, U.S.A.). The purity of PC was confirmed by thin-layer chromatography and it showed a single spot.

PC SUV Preparation By using a rotary evaporator and then a vacuum pump, $4.5 \mathrm{ml}$ of the $2 \%$ PC solution was dried. To the dried PC $5 \mathrm{ml}$ of the buffer were added so as to yield $c a .24 \mathrm{~mm}$ PC concentration and the mixture was vortexed to produce multilamellar vesicles. Then SUV were prepared by the sonication method as previously reported. ${ }^{10)}$

SUV size distribution was determined by a dynamic light scattering method using a submicron particle analyzer (Nicomp Model 380, Particle Sizing Systems, Santa Barbara, CA, U.S.A.), ${ }^{11)}$ and confirmed to be that the diameters of more than $90 \%$ of the vesicles were in the range of $20-30 \mathrm{~nm}$.

Phosphorus Determination The exact PC concentration in the SUV suspensions was calculated from phosphate analysis according to the phosphovanadomolybdate method. ${ }^{18)}$

Measurements of Absorption and Second Derivative Spectra To each of several $10 \mathrm{ml}$ volumetric flasks, an appropriate amount of phenothiazine stock solution was added so that the final drug concentration in the sample solutions became $15 \mu \mathrm{M}$. Then suitable amounts of the SUV suspensions were added to the flasks, and the buffer was further added to volume. Each flask was shaken for a short time and incubated at a given temperature in a temperature-regulated water bath for $30 \mathrm{~min}$. Then an absorption spectrum of the sample solution with a wavelength interval of $0.1 \mathrm{~nm}$ was measured against the reference solution by using a photodiode array spectrophotometer (Shimadzu MultiSpec-1500) equipped with a temperature-regulated cell holder in a 1-cm light-pass length cuvette at the given temperature.

The second derivative spectra based on the Savitzky-Golay method ${ }^{19)}$ were calculated by a personal computer ${ }^{20)}$ The cubic polynomial convolution of 17 points and the wavelength interval $(\Delta \lambda)$ of $0.5 \mathrm{~nm}$ was used in the calculation.

\section{Results and Discussion}

Absorption and Second Derivative Spectra The absorption spectra of TFPZ in the sample solutions containing various amounts of SUV at $37^{\circ} \mathrm{C}$ are shown in Fig. 2 as an example. The absorption maximum of TFPZ exhibits a bathocromic shift according to the increase in PC concentration indicating the partition of TFPZ to the PC bilayer of SUV, but any isosbestic point cannot be observed. It is obvious that the counterbalance of the background signals of SUV in the sample and reference beams is incomplete, despite the fact that the solutions in the sample and reference cuvettes were prepared to contain the same amount of SUV.

The second derivative spectra calculated from the absorption spectra in Fig. 2 are shown in Fig. 3. Similarly to Fig. 2,<smiles>[R1]CCCN1c2ccccc2Sc2ccc([R])cc21</smiles>

\begin{tabular}{|c|c|c|}
\hline Phenothiazine & $\mathrm{R}_{1}$ & $\mathrm{R}_{2}$ \\
\hline $\mathrm{TFPZ}$ & $-\mathrm{CF}_{3}$ & $-\mathrm{N}\left(\mathrm{CH}_{3}\right)_{2}$ \\
\hline $\mathrm{TFZ}$ & $-\mathrm{CF}_{3}$ & $-\mathrm{N}\left(\mathrm{CH}_{3}\right)_{2}$ \\
\hline $\mathrm{CPZ}$ & $-\mathrm{Cl}$ & $-\mathrm{N}\left(\mathrm{CH}_{3}\right)_{2}$ \\
\hline $\mathrm{PZ}$ & $-\mathrm{H}$ & $\mathrm{CH}_{3}$ \\
\hline
\end{tabular}

Fig. 1. The Chemical Structures of Phenothiazine Drugs Studied

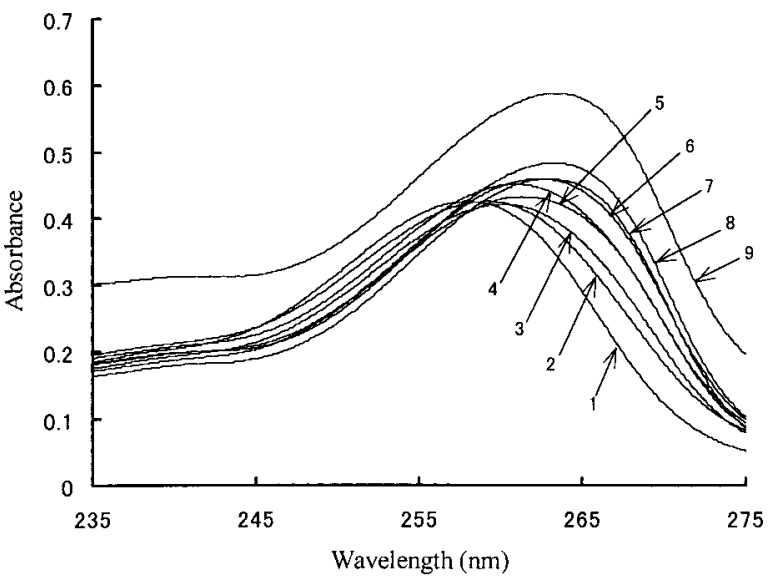

Fig. 2. Absorption Spectra of $15 \mu \mathrm{M}$ TFPZ in Hepes Buffer Solutions Varying Concentrations of SUV at $37^{\circ} \mathrm{C}$

PC concentration (m)): (1), 0; (2), 0.03; (3), 0.05; (4), 0.08; (5), 0.11; (6), 0.16; (7), $0.24 ;(8), 0.32 ;(9), 0.43$.

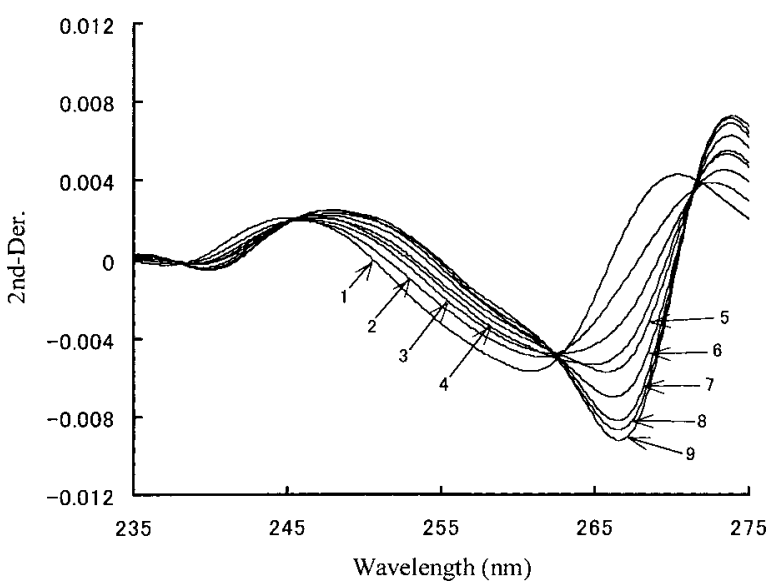

Fig. 3. Second Derivative Spectra of TFPZ Calculated from the Absorption Spectra in Fig. 2

The numbers in the figure are the same as in Fig. 2. 
a bathocromic shift is observed with an increase in the PC concentration. Three derivative isosbestic points are clearly observed in Fig. 3. The existence of the derivative isosbestic points indicates that the influences of the residual background signal of SUV are entirely eliminated in the second derivative spectra, and that TFPZ exists in two states, ${ }^{21)}$ i.e. in the bulk water and the PC bilayer of SUV. Similar results were obtained for TFZ, CPZ and PZ.

Calculated $\boldsymbol{K}_{\mathbf{p}}$ Values The $\Delta D$ values for TFPZ, TFZ, $\mathrm{CPZ}$ and PZ were obtained from the derivative values at the wavelength of 266, 265, 262 and $258 \mathrm{~nm}$, respectively. Using the $\Delta D$ values, the $K_{\mathrm{p}}$ and $\Delta D_{\max }$ values of the each phenothiazine drug at each temperature were calculated. The calculated $K_{\mathrm{p}}$ values are summarized in Table 1 and their R.S.D of below $10 \%$ confirms a good precision of the second derivative method.

In Fig. 4, the fractions of TFPZ partitioned in the PC bilayer of SUV at several temperatures are shown as a plot of the $\Delta D / \Delta D_{\max }$ values versus $\mathrm{PC}$ concentration. Solid lines represent theoretical curves calculated from Eq. 2 using the obtained $K_{\mathrm{p}}$ and $\Delta D_{\max }$ values. Each experimental value shows a good fit with the calculated curves, indicating accuracy of the $K_{\mathrm{p}}$ values obtained. The $K_{\mathrm{p}}$ values of TFZ, CPZ and $\mathrm{PZ}$ also showed similar results.

Temperature Dependence of Partitioning The thermodynamic parameters for the phenothiazine partitioning from bulk water phase to the PC bilayer of SUV were calculated from van't Hoff analysis based on the temperature dependence of $K_{\mathrm{p}}$ value, and the results were shown in Fig. 5 and

Table 1. The Temperature Dependence of $K_{\mathrm{p}} \mathrm{s}$ of Phenothiazine Drugs between PC SUV and Buffer (pH 7.4)

\begin{tabular}{ccccc}
\hline \hline \multirow{2}{*}{$\begin{array}{c}\text { Temperature } \\
\left({ }^{\circ} \mathrm{C}\right)\end{array}$} & \multicolumn{4}{c}{$K_{\mathrm{p}}\left(\times 10^{-5}\right)^{a)}$} \\
\cline { 2 - 5 } & $\mathrm{TFPZ}$ & $\mathrm{TFZ}$ & $\mathrm{CPZ}$ & $\mathrm{PZ}$ \\
\hline 10 & $6.02 \pm 0.12$ & $3.80 \pm 0.18$ & $3.18 \pm 0.09$ & $1.50 \pm 0.14$ \\
20 & $5.77 \pm 0.12$ & $3.55 \pm 0.21$ & $2.80 \pm 0.03$ & $1.18 \pm 0.09$ \\
30 & $5.32 \pm 0.26$ & $3.27 \pm 0.10$ & $2.36 \pm 0.09$ & $0.82 \pm 0.07$ \\
37 & $5.20 \pm 0.20$ & $3.41 \pm 0.30$ & $2.37 \pm 0.02$ & $0.79 \pm 0.03$ \\
40 & $5.01 \pm 0.38$ & $2.94 \pm 0.08$ & $2.10 \pm 0.05$ & $0.69 \pm 0.04$ \\
\hline
\end{tabular}

a) Each value is expressed as the mean \pm S.D. $(n=3)$.

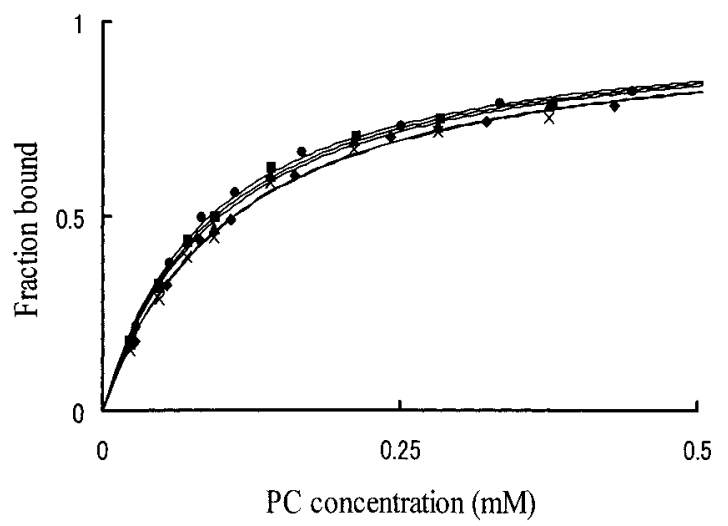

Fig. 4. Fraction of TFPZ in the PC Bilayer of SUV as a Function of PC Concentration at Different Temperatures

The solid lines show the theoretical curves calculated from Eq. 2 using the experimental values of $K_{\mathrm{p}}$ and $\Delta D_{\max }$. The symbols are the experimental values: $(\bullet) 10$, (ם) $20,(\boldsymbol{\Lambda}) 30,(\bullet) 37,(\times) 40^{\circ} \mathrm{C}$.
Table 2. As seen in Fig. 5, the van't Hoff plot for each phenothiazine drug revealed a linear line with a positive slope. The positive slopes, i.e., negative $\Delta H_{\mathrm{w} \rightarrow 1}$ values show that the partitioning of phenothiazine drugs is an exothermic process and it can be considered that the electrostatic interaction, positively charged alkyl amino groups of the phenothiazine drugs and negatively charged phosphate groups on the surface of PC SUV, partly contributes to the partitioning.

Meanwhile, the $\Delta S_{\mathrm{w} \rightarrow 1}$ values are positive as seen in Table 2. Thus, being associated with negative $\Delta H_{\mathrm{w} \rightarrow 1}$ and positive $\Delta S_{\mathrm{w} \rightarrow 1}$, the partitioning of phenothiazine drugs into PC bilayer membranes is considered to be an entropy/enthalpy-driven reaction. ${ }^{22)}$ This result is consistent with the result of a thermodynamic study on the interactions of phenothiazine drugs with erythrocyte ghost membranes performed by a microcalorimetry. ${ }^{23)}$ Therefore, it has been confirmed that SUV is far preferable for a biomembranes model than multilamellar vesicles also in thermodynamic studies. ${ }^{1)}$

The $\Delta S_{\mathrm{w} \rightarrow 1}$ value was enhanced by introducing halogen atom(s) at position $\mathrm{C}-2$ on the phenothiazine ring $(\mathrm{H}<$ $\mathrm{Cl}<\mathrm{CF}_{3}$ ) as seen in Table 2. This can be considered as that $\Delta S_{\mathrm{w} \rightarrow 1}$ reflects the increase of motions of acyl chain region in the PC bilayer membranes, i.e., the insertion of phenothiazine ring as a result of the partitioning will give some perturbations to the motions of acyl chain regions and the extent of the perturbation will be in order of $\mathrm{H}<\mathrm{Cl}<\mathrm{CF}_{3}$. The $\Delta S_{\mathrm{w} \rightarrow 1}$ values of TFPZ and TFZ show similarly about $60 \mathrm{~J} \cdot \mathrm{mol}^{-1} \mathrm{~K}^{-1}$, though the $K_{\mathrm{p}}$ values were considerably different, which can be explained that both TFPZ and TFZ have a trifluoromethyl group and thus have similar interactions with the acyl chain regions of the PC bilayer membranes.

From enthalpy-pentropy correlation analysis of plotting $\Delta S_{\mathrm{w} \rightarrow 1}$ against $\Delta H_{\mathrm{w} \rightarrow 1}$ as in Fig. 6, a good linear relationship is confirmed by an excellent correlation coefficient $(r=$

Table 2. The Thermodynamics of Partitioning of Phenothiazine Drugs in PC SUV at $\mathrm{pH} 7.4$ and $37^{\circ} \mathrm{C}$

\begin{tabular}{cccc}
\hline \hline Phenothiazine & $\begin{array}{c}\Delta H_{\mathrm{w} \rightarrow 1} \\
(\mathrm{~kJ} / \mathrm{mol})\end{array}$ & $\begin{array}{c}\Delta G_{\mathrm{w} \rightarrow 1} \\
(\mathrm{~kJ} / \mathrm{mol})\end{array}$ & $\begin{array}{c}\Delta S_{\mathrm{w} \rightarrow 1} \\
(\mathrm{~J} / \mathrm{mol} \cdot \mathrm{K})\end{array}$ \\
\hline TFPZ & -4.50 & -24.29 & 63.82 \\
TFZ & -5.01 & -23.20 & 58.69 \\
CPZ & -9.48 & -22.26 & 41.23 \\
PZ & -18.71 & -19.42 & 2.31 \\
\hline
\end{tabular}

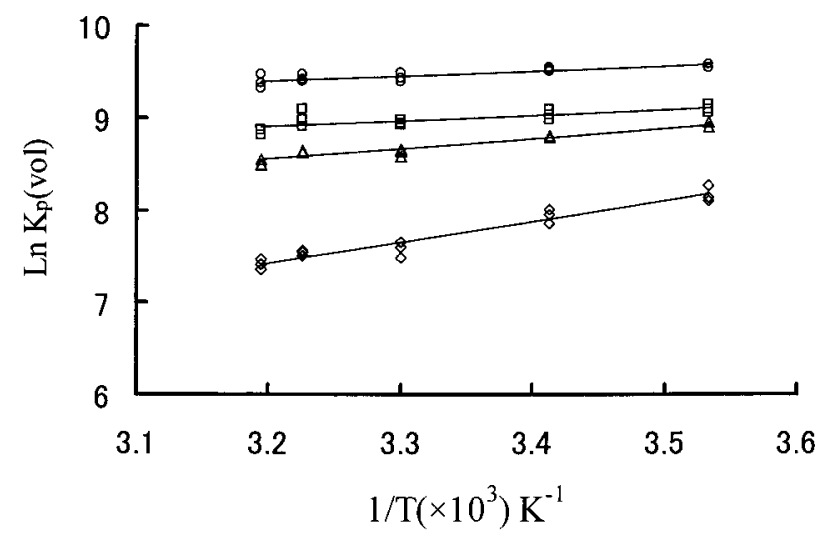

Fig. 5. Van’t Hoff Plots of $(\bigcirc)$ TFPZ, $(\square)$ TFZ, $(\triangle)$ CPZ and $(\diamond) \mathrm{PZ}$ in PC SUV at $\mathrm{pH} 7.4$ 


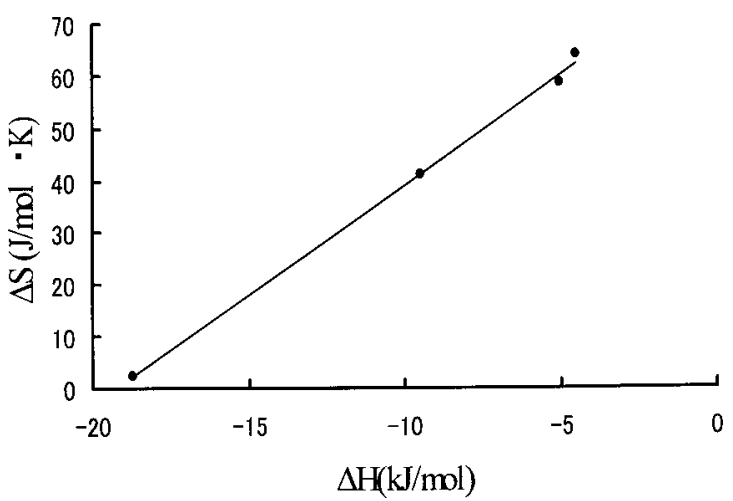

Fig. 6. Correlation between $\Delta H_{\mathrm{w} \rightarrow 1}$ and $\Delta S_{\mathrm{w} \rightarrow \mathrm{l}}$ for the Phenothiazne Drugs Partitioning into PC Bilayer of SUV

0.999), showing strong entropy-enthalpy compensation. The result also indicates that the phenothiazine drugs studied have an identical interaction mechanism with the PC bilayer membranes. $^{24)}$

In conclusion, our results give some useful information for the consideration with respect to the phenothiazine drug transport process into biomembranes. Also, it can be emphasized that the second-derivative spectrophotometric method can enable to use SUV in thermodynamic studies of the drug interactions with lipid bilayer membranes without any perturbations on the equilibrium state and temperature of the sample solutions. It is also elucidated that to use SUV as a biomembrane model is far preferable than MLV which is forced to use in the methods employing separation procedures.

\section{References}

1) Ahmed A. M. S., Farah F. H., Kellaway I. W., Pharm. Res., 1, 119-
124 (1985).

2) Betageri G. V., Rogers J. A., Int. J. Pharmaceut., 36, 165-173 (1987).

3) Betageri G. V., Rogers J. A., J. Pharm. Phrmacol., 45, 931-933 (1993).

4) Betageri G. V., Nayernama A., Habib M. J., Int'l. J. of Pharm. Advances, 1, 310-319 (1996).

5) Keller M., Kerth A., Blume A., Biochim. Biophys. Acta, 1326, 178 198 (1997).

6) Go M. L., Ngian T. L., Chem. Pharm. Bull., 45, 2055-2060 (1997).

7) Choi Y. W., Rogers J. A., Pharm. Res., 7, 508-512 (1990).

8) Fujiwara H., Da Y. Z., Ito K., Takagi T., Nishioka Y., Bull. Chem. Soc. Jpn., 64, 3707-3712 (1991).

9) Rogers J. A., Choi Y. W., Pharm. Res., 10, 913-917 (1993).

10) Kitamura K., Imayoshi N., Goto T., Shiro H., Mano T., Nakai Y., Anal. Chim. Acta, 304, 101-106 (1995).

11) Takegami S., Kitamura K., Kitade T., Hasegawa K., Nishihira A., J. Coll. Interf. Sci., 220, 81-87 (1999).

12) Kitamura K., Takenaka M., Yoshida S., Ito M., Nakamura Y., Hozumi K., Anal. Chim. Acta, 242, 131-135 (1991).

13) Kitamura K., Kitade T., Mabuchi M., Mizuochi M., Goto K., Bunseki Kagaku, 42, 581—585 (1993).

14) Bondy B., Remien J., Life Sci., 28, 441-449 (1981).

15) Luxnat M., Galla H. J., Biochim. Biophys. Acta, 856, 274-282 (1986).

16) Zachowski A., Durand P., Biochim. Biophys. Acta, 937, 411-416 (1988)

17) Welti R., Mullikin L. J., Yoshimura T., Helmkamp G. M., Jr., Biochemistry, 23, 6086-6091 (1984).

18) Christopher A. J., Fennell T. R. F. W., Microchem. J., 12, 593-605 (1967).

19) Savitzky A., Golay M. J. E., Anal. Chem., 36, 1627-1639 (1964).

20) Kitamura K., Hozumi K., Anal. Chim. Acta, 172, 111-118 (1985).

21) Connors K. A., "Binding Constants," John Wiley \& Sons, New York, 1987, pp. 142-147.

22) Pliska V., Testa B., Van de Waterbeemd H., "Lipophilicity in Drug Action and Toxicology," VCH, Germany, 1996, pp. 285-290.

23) Aki H., Yamamoto M., J. Pharm. Pharmacol., 42, 637-641 (1990).

24) Leffler J. E., Grunwald E., "Rates and Equilibria of Organic Reactions," John Wiley \& Sons, New York, 1963, pp. 128-314. 